\title{
A Simulation Study of OBDD-Based Proper Splitting Strategies for Power Systems Under Consideration of Transient Stability
}

\author{
Kai Sun, Da-Zhong Zheng, and Qiang Lu, Fellow, IEEE
}

\begin{abstract}
System splitting, also called controlled system islanding, can effectively prevent blackouts. Following the OBDD-based three-phase method given in [3] for proper splitting strategies, which satisfy necessary steady-state constraints, this paper studies the feasibility of the proper splitting strategies by means of power system transient simulations on the IEEE 118-bus system. Simulation results show that a considerable proportion of proper splitting strategies can successfully split the power system into stable islands. Furthermore, considering a general knowledge that a controlled power system can easily maintain its stability after a small disturbance, this paper presents "threshold value constraint" to restrict the degrees of the disturbances caused by proper splitting strategies. An approach is proposed to select threshold values for the constraint. Further simulation results show that by checking the constraint for proper splitting strategies, feasible splitting strategies can be found, which can successfully split the system into islands satisfying transient stability constraints.
\end{abstract}

Index Terms-OBDD, simulation study, splitting strategy, system islanding, system splitting, threshold value constraint, transient stability.

\section{NOMENCLATURE}

ASDP Acceptable maximal splitting delay period, which is defined in Section IV-A.

OBDD Ordered binary decision diagram. Refer to [1] for details.

PBC The constraint that the generation and load in each island are balanced after system splitting. Refer to [2] for details.

RLC The constraint that all transmission lines and other transmission devices must not be loaded above their transmission capacity limits. Refer to [2] for details.

SDP Splitting delay period, the time from clearing faults to splitting the power system, which is defined in Section IV-A.

SSC The constraint that asynchronous groups of generators are separated. Refer to [2] for details.

TVC Threshold value constraint, which is proposed in Section VI-C.

Manuscript received May 8, 2004. This work was supported in part by NSFC (Grant 60074012 and Grant 60274011) and in part by the National Fundamental Research Funds under Grant G1998020310 and Tsinghua University project. Paper no. TPWRS-00005-2004.

K. Sun and D.-Z. Zheng are with the Center for Intelligent and Networked Systems, Department of Automation, Tsinghua University, Beijing 100084, China (e-mail: sunkai95@tsinghua.org.cn).

Q. Lu is with the Department of Electrical Engineering, Tsinghua University, Beijing 100084, China.

Digital Object Identifier 10.1109/TPWRS.2004.841239

\section{INTRODUCTION}

YSTEM splitting, also called controlled system islanding, $\boldsymbol{N}$ is to open selected transmission lines to split a power system into islands of load with matched generation. When faults cause loss of synchronism of generators and emergency control actions cannot keep the integrity of the power network, system splitting is an effective measure to prevent a blackout. In general, a splitting strategy must be online given (within several seconds or even less than $1 \mathrm{~s}$ ) when the power system needs to be split into islands. However, for a large-scale power system, it is quite difficult to online find a splitting strategy even only satisfying necessary steady-state constraints since a combinatorial explosion of its strategy space is unavoidable. For example, for the IEEE 118-bus system with 186 lines, the strategy space for system splitting includes $2^{186} \approx 9.8 \times 10^{55}$ possible choices! Applying OBDD (ordered binary decision diagram) representation [1], [3] proposes a three-phase method (for short, the OBDD method) to online search for splitting strategies for large-scale power systems. The splitting strategies all satisfy three kinds of constraints, SSC, PBC, and RLC, and are called "proper splitting strategies" in [2]. SSC denotes that asynchronous groups of generators are separated, $\mathrm{PBC}$ denotes that in each island, generation and load are matched with an acceptable error, and RLC denotes that transmission lines and other transmission devices must not be loaded above their transmission capacity limits. The simulation results in [3] demonstrate that the OBDD method is effective to online search for proper splitting strategies. Since the OBDD method mainly considers steady-state constraints, every proper splitting strategy, in fact, decides an acceptable steady-state operating point of the power system following system splitting. However, [3] does not analyze the transient stability of the power system after system splitting is performed, and does not answer whether a proper splitting strategy can make the power system reach an acceptable steady-state operating point.

In this paper, the feasibility of the proper splitting strategies is studied by means of power system transient simulations on the IEEE 118-bus system. Simulation results show that among the proper splitting strategies given by the OBDD method, a considerable proportion of strategies can successfully split the power system into stable islands. In other words, they are feasible splitting strategies. Then, this paper studies how to find feasible splitting strategies. Because a feasible splitting strategy generally needs to be given within several seconds or even less than 
$1 \mathrm{~s}$, conventional transient stability analysis methods cannot effectively find a feasible splitting strategy within such a short time. In order to quickly find feasible splitting strategies, this paper explores a novel way, which is based on the following two considerations: system splitting according to different splitting strategies would bring different degrees of power-flow disturbances; it is a general knowledge that a controlled power system can easily maintain its stability under a small disturbance. Thus, "threshold value constraint" (TVC) is presented to restrict the degrees of the disturbances caused by proper splitting strategies. Then, an approach is proposed to offline select the threshold values for TVC. Further simulation results show that feasible splitting strategies can quickly be found by checking TVC for proper splitting strategies to successfully split the power system into islands satisfying transient stability constraints.

In the rest of the paper, Section II reviews the OBDD method. Section III introduces the test power system and its models. Main results of this paper are given in Section IV. Finally, Section $\mathrm{V}$ provides some conclusions.

\section{A REVIEW FOR THE OBDD METHOD}

The OBDD-based three-phase method (for short, the OBDD method) is proposed in [3] and can quickly find enough proper splitting strategies for a large-scale power system. Its flow chart is shown in Fig. 1. The function of its Phase-1 is to initialize parameters and reduce the original complicated power network by two simplification measures [3] based on graph theory and the characteristics of the power network. The function of its Phase-2 is to search for splitting strategies satisfying SSC and PBC by OBDD-based algorithms. Finally, its Phase-3 checks RLC for the splitting strategies by power-flow calculations and gives the strategies satisfying RLC as proper splitting strategies. The reduction process of strategy space is also shown in Fig. 1. After the Phase-1, the original strategy space is reduced to a smaller elliptic region (called "searching space"). Then, the Phase-2 and Phase- 3 are both carried out in "searching space." Their results are respectively the intersections of the ellipse and the two rectangles in Fig. 1.

Reference [3] tests the performance of the OBDD method on the IEEE 118-bus system by means of PC with Pentium IV 1.4-GHz processor and 256-MB RAM. In order to shorten the online searching time for proper splitting strategies, all tasks of the OBDD method are separated into three time layers, namely Offline Layer, Period Layer and Online Layer. Only the tasks in Online Layer must be completed online. Simulation results show that the online searching time for all splitting strategies satisfying SSC and PBC in "searching space" is about 0.1 $\mathrm{s}$ and the time for checking $R L C$ for a strategy is less than $0.007 \mathrm{~s}$. Furthermore, many tasks of the method (e.g., building the OBDD of PBC and checking RLC) can be performed on parallel processors to further reduce the online searching time. Thus, the OBDD method can be more practical if efficient concurrent technology is applied, and advanced computer systems with high-performance hardware and professional software are adopted.

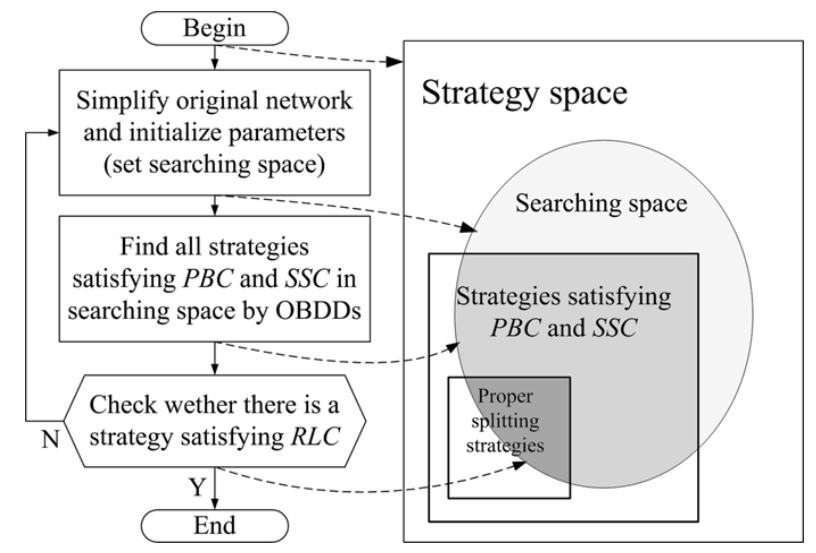

Fig. 1. Flowchart of the OBDD method.

\section{The Test Power System And ITS Models}

\section{A. The Test Power System}

The IEEE 118-bus system is selected as the test power system in this paper due to the following considerations. A large-scale power system may own thousands of buses. However, when we study its splitting strategies, it is advisable to mainly consider its backbone grid, e.g., its $500 \mathrm{kV}$ backbone grid. In the backbone grid, the buses connecting main power plants are regarded as generator buses and all the other buses are regarded as load buses; all the buses not in the backbone grid are ignored in searching for splitting strategies. In order to guarantee that a splitting strategy facing the backbone grid can split the whole power system into islands, some fixed splitting points below the backbone grid should be selected in advance. The fixed splitting points can be set according to real geographic regions in the power network. As soon as the backbone grid is split, some of the fixed splitting points will directly be cut off. Therefore, the backbone grid can be regarded as a simplified network of the power network. In fact, most of real large-scale power systems' backbone grids are simpler than the IEEE 118-bus system. For instance, a large-scale power system in China covers 5 provinces and spans longer than $1500 \mathrm{~km}$, but its $500-\mathrm{kV}$ backbone grid only owns no more than 60 buses.

\section{B. Graph-Model}

Reference [2] uses an undirected, connected and nodeweighted graph $G(V, E, W)$ (called "graph-model") to depict a $n$-bus power network, where $V=\left\{v_{1}, \ldots, v_{n}\right\}$ is the node set, $W=\left\{w_{1}, \ldots, w_{n}\right\}$ is the node weight set and $E$ is the edge set. Elements $v_{i}$ and $w_{i}$ respectively correspond to bus $i$ and its real power (i.e., the real generation power minus the real load power of bus $i$ ). If there is a transmission line connecting bus $i$ and bus $j, E$ must have an element $e_{i j}$ (assume $i<j$ ). Thus, a splitting strategy can be denoted by an edge set $S \subset E$. The graph-model is a useful power system model for studying system splitting. The graph-model of the IEEE 118-bus system is shown in Fig. 2, where white dots are called "generator nodes" denoting generator buses, which are connected with generators, and black dots are called "load nodes" denoting the other buses. 


\section{Transient Simulation Model}

In this paper, a simple but representative power system model is used for power system transient simulations. Firstly, generator $i$ is represented by the classical model with an imaginary constant EMF $E_{i}$ behind the direct axis transient reactance $x_{d, i}^{\prime}$ [4]. Moreover, a simplified control loop of each generator is considered as is shown in Fig. 3. The reference value of each speed governor (denoted by $P_{\text {ref }, i}$ ) is assumed to be constant. Thus, the power system model can be described by the following equations:

$$
\begin{aligned}
\dot{\delta}_{i}= & \omega_{0} \cdot \Delta \omega_{i}=\omega_{0} \cdot\left(\omega_{i}-1\right) \\
M_{i} \Delta \dot{\omega}_{i}= & P_{m, i}-P_{e, i}-D_{i} \Delta \omega_{i} \quad i=1, \ldots, N_{G} \\
P_{e, i}= & E_{i}^{2} G_{i i}+\sum_{k=1, k \neq i}^{n} E_{i} E_{k}\left[B_{i k} \sin \left(\delta_{i}-\delta_{k}\right)\right. \\
& \left.+G_{i k} \cos \left(\delta_{i}-\delta_{k}\right)\right] \\
Y_{i k}= & G_{i k}+j B_{i k} \\
T_{G, i} \Delta \dot{a}_{i}= & -\Delta a_{i}+K_{G, i}\left(\Delta P_{\mathrm{ref}, i}-\frac{\Delta \omega_{i}}{R_{i}}\right) \\
T_{T, i} \Delta \dot{P}_{m, i}= & -\Delta P_{m, i}+K_{T, i} \Delta a_{i}
\end{aligned}
$$

where $N_{G}$ is the number of generators in the power system and $\omega_{0}=2 \pi f_{0}$ is the reference speed, where $f_{0}=60 \mathrm{~Hz}$ is the rating frequency. For generator $i, \delta_{i}$ is the mechanical rotor angle in $\mathrm{rad}, \omega_{i}$ is the mechanical rotor speed in per unit (p.u.), $M_{i}$ is the inertia constant in s, $D_{i}$ is the damping coefficient, $P_{e, i}$ is the electric power output in p.u., and $P_{m, i}$ is the mechanic power input in p.u. $Y$ is the network's admittance matrix (all loads have been represented by constant impedances, and all buses have been eliminated). Its element $Y_{i k}$ has a real part $G_{i k}$ and an imaginary part $B_{i k}$.

In Fig. 3, $\Delta P_{\text {ref, } i}$ is the increment of $P_{\text {ref, } i}$ in p.u. Since $P_{\text {ref, }, i}$ is constant, $\Delta P_{\text {ref }, i}=0 . \Delta P_{m, i}=P_{m, i}-P_{m, i, 0}$, where $P_{m, i, 0}$ is the initial mechanical power in p.u. $\Delta a_{i}$ (in pu) is the increment of the valve opening $a_{i} . \Delta a_{M, i}$ and $\Delta a_{m, i}$ are respectively its upper limit and lower limit, which are determined by the initial valve opening and the scope of $a_{i} . K_{G, i}$ and $K_{T, i}$ are respectively the gains of the speed-governor and turbine, which both equal 1 if all variables are expressed in p.u. $T_{G, i}$ and $T_{T, i}$ are, respectively, the time constants of the speed-governor and turbine. Finally, $R_{i}$ is the regulation constant of generator $i$.

\section{Simulation Data}

Table I gives generator data, which are selected according to the typical generator data in [5]. The use of symmetrical components [7] for fault analysis allows any type of fault to be represented in the positive sequence network by a fault shunt reactance connected between the point of the fault and the neutral. In order to calculate the fault shunt reactance, the negative-sequence impedances of generators are necessary (refer to [7] and [8] for details). In Table $\mathrm{I}, x_{-, i}$ is the magnitude of the negative-sequence impedance of generator $i$. Moreover, for all generators, let $K_{G, i}=K_{T, i}=1, T_{T, i}=0.3 \mathrm{~s}$, $T_{G, i}=0.2 \mathrm{~s}, R_{i}=0.05$, and $a_{i} \geq 0\left(i=1 \sim N_{G}\right)$.

The network data used in simulations are standard (see [6]) except transmission line capacities, which are all assumed to

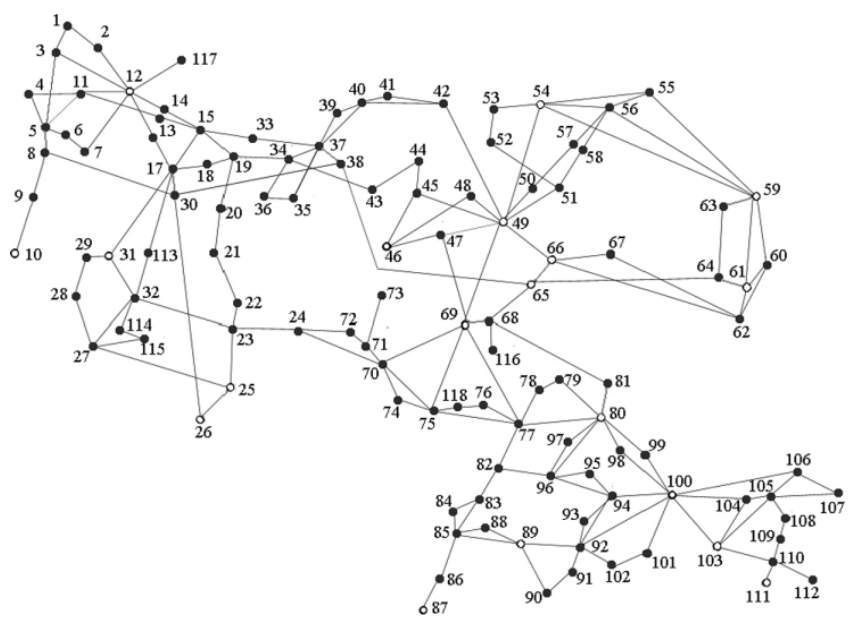

Fig. 2. Graph-model of the IEEE 118-bus system.

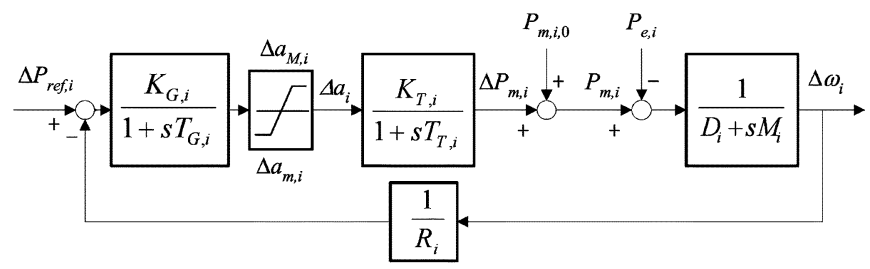

Fig. 3. Simplified control loop of generator $i$.

TABLE I

GENERATOR DATA

\begin{tabular}{c|c|c|c|c}
\hline Bus No. & $M_{i}(\mathrm{~s})$ & $D_{i}(\mathrm{pu})$ & $x^{\prime}{ }_{d, i}(\mathrm{pu})$ & $x_{-, i}(\mathrm{pu})$ \\
\hline \hline 10 & 5.66 & 3.0 & 0.059 & 0.039 \\
\hline 12 & 9.97 & 3.0 & 0.220 & 0.095 \\
\hline 25 & 8.24 & 3.0 & 0.139 & 0.106 \\
\hline 26 & 6.01 & 3.0 & 0.096 & 0.052 \\
\hline 31 & 12.37 & 3.0 & 0.247 & 0.113 \\
\hline 46 & 12.37 & 3.0 & 0.247 & 0.113 \\
\hline 49 & 8.24 & 3.0 & 0.139 & 0.106 \\
\hline 54 & 9.97 & 3.0 & 0.220 & 0.095 \\
\hline 59 & 7.93 & 3.0 & 0.153 & 0.072 \\
\hline 61 & 7.93 & 3.0 & 0.153 & 0.072 \\
\hline 65 & 7.41 & 3.0 & 0.067 & 0.055 \\
\hline 66 & 7.41 & 3.0 & 0.067 & 0.055 \\
\hline 69 & 5.26 & 3.0 & 0.053 & 0.030 \\
\hline 80 & 5.26 & 3.0 & 0.053 & 0.030 \\
\hline 87 & 12.37 & 3.0 & 0.247 & 0.113 \\
\hline 89 & 4.64 & 3.0 & 0.047 & 0.036 \\
\hline 100 & 8.26 & 3.0 & 0.095 & 0.052 \\
\hline 103 & 9.97 & 3.0 & 0.220 & 0.095 \\
\hline 111 & 9.97 & 3.0 & 0.220 & 0.095 \\
\hline
\end{tabular}

be $900 \mathrm{MW}$, since most transmission lines in an actual power system have enough transmission capacities.

\section{MAin Results BASEd on Simulations}

\section{A. Feasible Splitting Strategies}

Assume that the whole system becomes asynchronous after faults are cleared by local relays. Before system splitting is performed, it must take some time to finish some necessary tasks, e.g., detecting asynchronous groups of generators, searching for splitting strategies and executing other associated operations. In 
this paper, the time from clearing faults to splitting the power system is called "splitting delay period", denoted by SDP.

Then, "feasible splitting strategy" is defined as follows. A proper splitting strategy is called a "feasible splitting strategy" if it splits the power system into islands with $\operatorname{SDP} \geq \varepsilon$, where $\varepsilon$ is a small positive number, and the following two conditions are satisfied in each island.

1) All generators can keep synchronous within time $T_{S}$, which is enough to start up main emergency control actions.

2) Each bus voltage $V_{i}$ and generator frequency $f_{j}$ can respectively be stabilized near the rating voltage $V_{0}$ and the rating frequency $f_{0}$ with offsets not more than $\Delta V$ and $\Delta f$.

Remark: Since SDP is unlikely to be too short, a small positive number $\varepsilon$ is set, which is assumed to be $0.05 \mathrm{~s}$ in the simulations below. Moreover, in this paper, the simulation model given by (1)-(6) is quite simple, and most actual emergency control actions are not considered. Therefore, we reasonably relax the demands on $T_{S}, \Delta V$, and $\Delta f$ in the simulations below, and let $T_{S}=3 \mathrm{~s}, \Delta V=0.1 \cdot V_{0}$, and $\Delta f=0.1 \cdot f_{0}$. In actual system splitting, emergency control actions are helpful to stabilize each island and regulate each bus voltage and generator frequency more close to $V_{0}$ and $f_{0}$, respectively.

Furthermore, for each feasible splitting strategy, the maximal SDP that makes all islands still satisfy the above two conditions can be estimated. It is called "acceptable maximal splitting delay period" (ASDP) of the feasible splitting strategy. Obviously, every ASDP $\geq \varepsilon$. Thus, if a feasible splitting strategy is applied, and its ASDP $\geq$ SDP, it must lead to a success system splitting and produce stable islands. Hence, ASDP is an important parameter of feasible splitting strategies and can reflect how easily a feasible splitting strategy can split the power system into stable islands.

Moreover, if a splitting strategy is not a feasible splitting strategy, it is called an "unfeasible splitting strategy" in this paper.

\section{B. A Case Study for the Feasibility of Proper Splitting Strategies}

Among the 19 generators of the IEEE 118-bus system, generators $12,31,46,54,87,103$, and 111 have much smaller capacities than the others and hence tripping the seven generators could not seriously affect the power balance of the whole power system. Therefore, in this paper, the seven generators are called "unimportant" generators and the others are called "important" generators. In order to increase the efficiency of the OBDD method, the following measures about "unimportant" generators are used in the simulations below.

1) In Phase-1, all the 7 "unimportant" generators are omitted and their corresponding seven generator nodes in the graph-model $G$ are regarded as load nodes. Consequently, a new graph-model (denoted by $G^{\prime}$ ) with 12 generator nodes is formed from $G$. Then, $G^{\prime}$ is reduced by the simplification measures of the Phase-1.

2) In the Phase-2, only "important" generators are considered in forming the Boolean expressions and the OBDD's of SSC and PBC. Therefore, the splitting strategies found by OBDD-based algorithms are only for $G^{\prime}$. Then, for each of the splitting strategy, all "unimportant" generators are considered: if an "unimportant" generator is asynchronous with the island including it, it will be directly tripped.

3) For each splitting strategy given by the Phase-2, the Phase- 3 calculates the power flow of the power network without the "unimportant" generators that have been tripped.

Then, a simulation study is arranged as follows. Suppose that at the time $t=0.0 \mathrm{~s}$, a three-phase fault occurs near bus 25 at line 23-25 in the IEEE 118-bus system and is cleared after local relays open the line at $t=0.17 \mathrm{~s}$. If no other emergency control action is executed, loss of synchronism of generators must happen. Fig. 4 gives some dynamic curves of generators to show the loss of synchronism. It can be observed that within a short time after the fault is cleared, generators $31,46,49,54,59,61$, $65,66,69,80,87,89,100,103$, and 111 have close rotor angles and frequencies, and generators $10,12,25$, and 26 also have close rotor angles and frequencies. That implies that all generators can be divided into two groups, namely $\{10,12,25,26\}$ and $\{31,46,49,54,59,61,65,66,69,80,87,89,100,103,111\}$.

Then, using the OBDD method and the above measures about "unimportant" generators, we can find proper splitting strategies to separate the two groups of generators. For this case, 8233 proper splitting strategies can quickly be found. In fact, the number of proper splitting strategies depends on the parameters in the OBDD method.

Among the proper splitting strategies, randomly select 500 strategies and check their feasibility by means of power system transient simulations. Finally, 291 (58.2\%) strategies are found to be feasible splitting strategies, and the other 209 (41.8\%) strategies are unfeasible splitting strategies. The ASDP's of the 291 feasible splitting strategies are found to belong to 8 separate open intervals as shown in Table II, where the numbers and percentages of corresponding feasible splitting strategies are also given.

It can easily be found from Table II that the ASDPs of about $95 \%$ feasible splitting strategies are between 0.39 and 0.44 s. For the 500 strategies, Fig. 5 plots the relation between SDP and the percentage of the proper splitting strategies that can lead to a successful system splitting (in other words, the two conditions in Sub-Section IV.A are satisfied in each island). From Fig. 5, if SDP $\leq 0.39 \mathrm{~s}$, more than $50 \%$ proper splitting strategies can lead to a successful system splitting.

Finally, Fig. 6 gives the dynamic curves of generators after the power system is split at $\mathrm{t}=0.57 \mathrm{~s}$ (i.e., $\mathrm{SDP}=0.4 \mathrm{~s}$ ) by a feasible splitting strategy whose ASDP $\approx 0.43 \mathrm{~s}$. The feasible splitting strategy trips an "unimportant" generator 31, so the "Group-I" and "Group-II" in Fig. 6 are respectively $\{10,12,25,26\}$ and $\{46,49,54,59,61,65,66,69,80,87,89,100,103\}$. It can be observed that the rotor angles, frequencies, and bus voltages of the other generators are all stable.

From the above simulation results, the following conclusions can be drawn. 


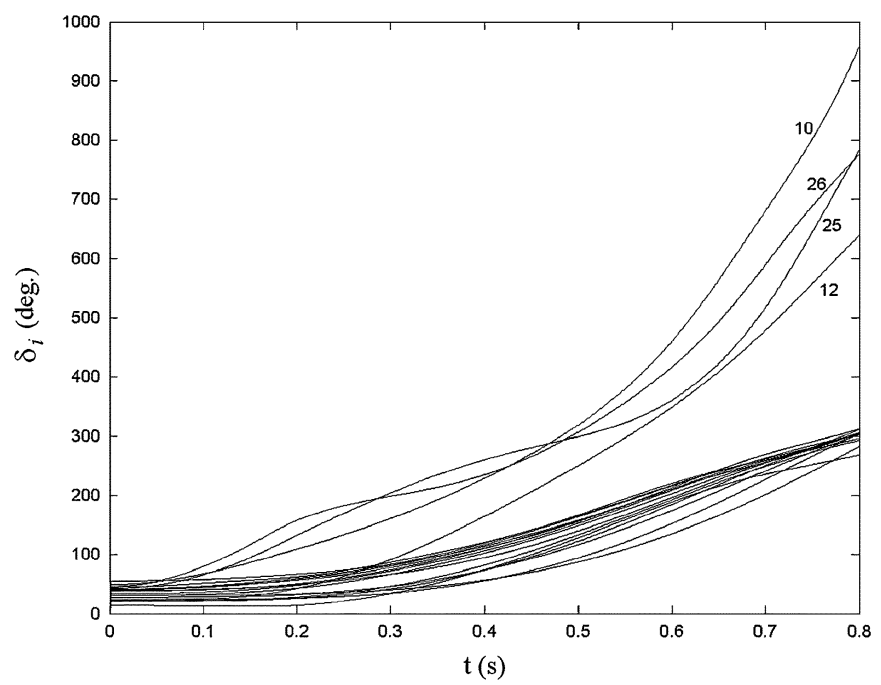

(a)

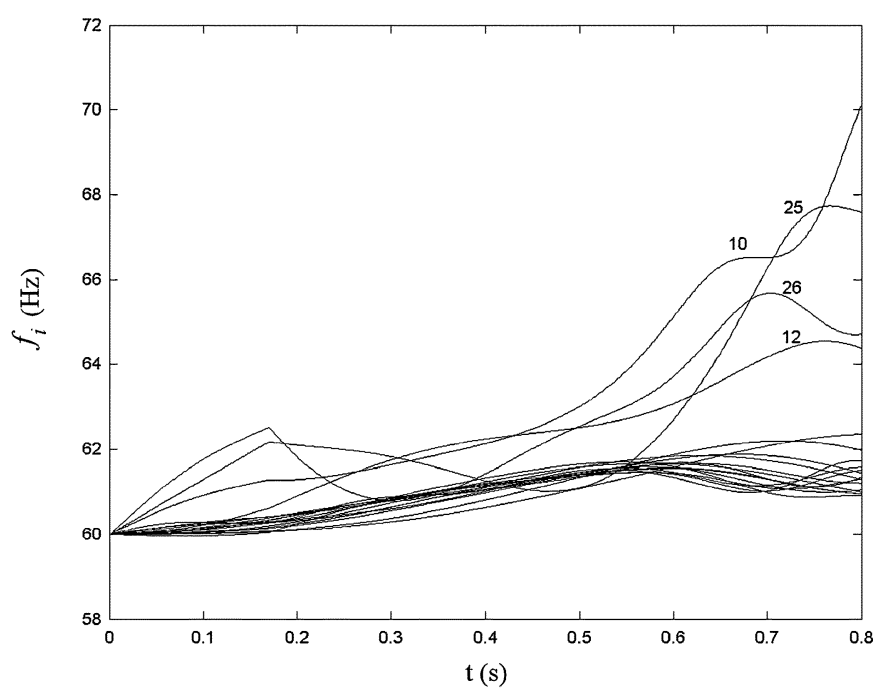

(b)

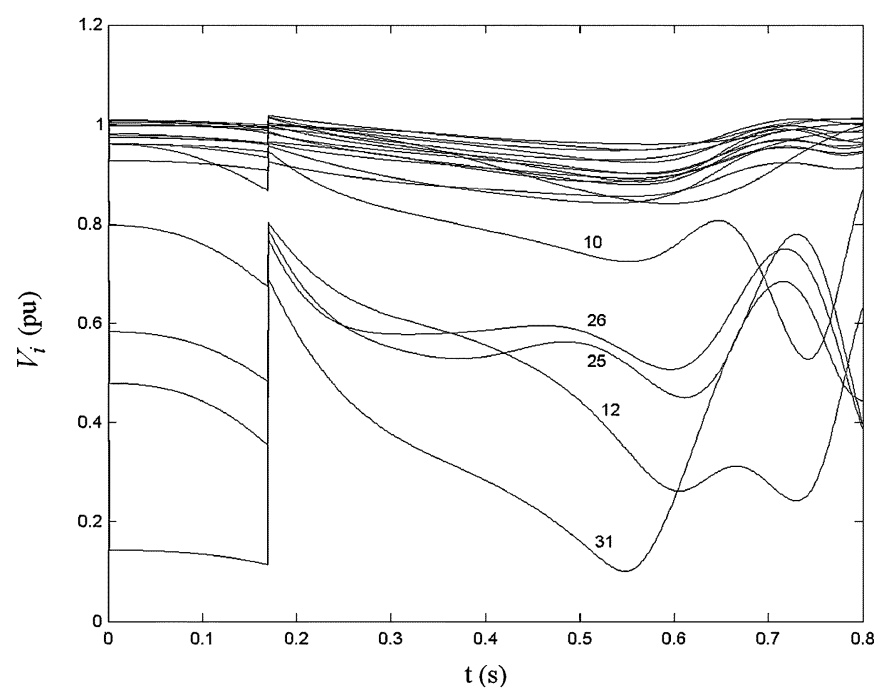

(c)

Fig. 4. Some dynamic curves of generators after a fault is cleared at line 23-25. (a) Angular rotor swings of all generators. (b) Frequencies of all generators. (c) Voltages of all generator buses.

1) Feasible splitting strategies exist among the proper splitting strategies given by the OBDD method, and their per-
TABLE II

ASDPS OF FEASIBLE SPLITTING STRATEGIES

\begin{tabular}{c|c|c|c}
\hline No. & $\begin{array}{c}\text { The open intervals } \\
\text { including } A S D P \text { s (s) }\end{array}$ & Number & Percentage (\%) \\
\hline \hline 1 & $(0.43,0.44)$ & 3 & 0.6 \\
\hline 2 & $(0.42,0.43)$ & 17 & 3.4 \\
\hline 3 & $(0.41,0.42)$ & 43 & 8.6 \\
\hline 4 & $(0.40,0.41)$ & 147 & 29.4 \\
\hline 5 & $(0.39,0.40)$ & 66 & 13.2 \\
\hline 6 & $(0.35,0.36)$ & 1 & 0.2 \\
\hline 7 & $(0.20,0.21)$ & 13 & 2.6 \\
\hline 8 & $(0.05,0.06)$ & 1 & 0.2 \\
\hline
\end{tabular}

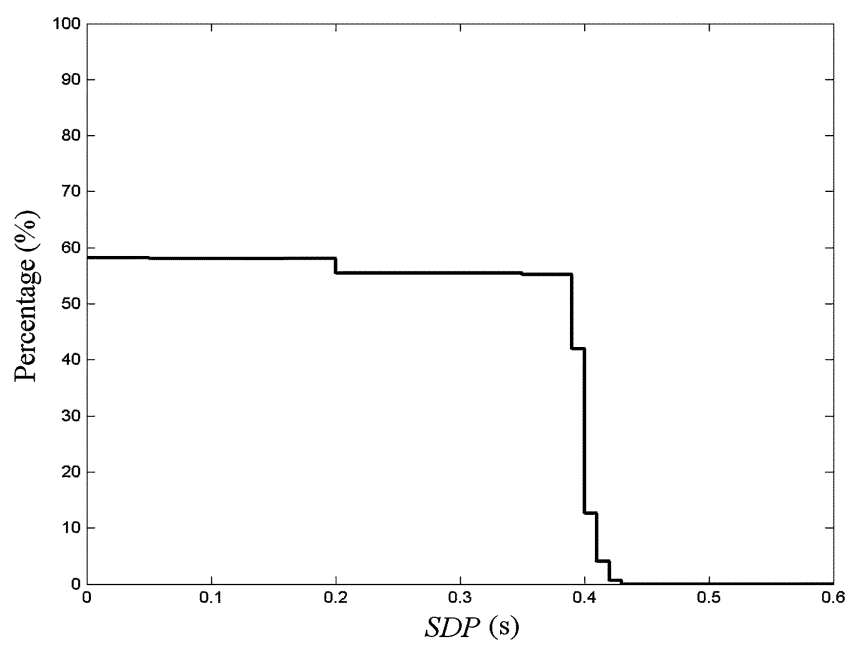

Fig. 5. Relation between SDP and the rate of the proper splitting strategies that can lead to a successful system splitting.

centage may be rather high (e.g., the percentage is higher than $50 \%$ in the above case).

2) The ASDP of a feasible splitting strategy is usually quite short. For example, in the above case, all ASDPs $<0.5 \mathrm{~s}$. Although the ASDP of a feasible splitting strategy may be prolonged with the aid of effective emergency control actions, it is not enough to analyze or test the transient stability of all islands by means of conventional transient stability analysis methods. Hence, it is difficult to online find a feasible splitting strategy by the conventional methods.

3) From the time performance of the OBDD method given in [3], the OBDD method is promising to online find enough proper splitting strategies within the ASDP's of most feasible splitting strategies if advanced computers are used. Thus, it is possible to develop a new method based on the OBDD method to online determine a feasible splitting strategy.

In the following subsection, a new constraint is introduced to indirectly check the transient stability of each island and find feasible splitting strategies from proper splitting strategies.

\section{Threshold Value Constraint and Threshold Value Strategies}

If a splitting strategy cuts too many lines or cuts some lines with large transmission real powers, a severe redistribution of the power flow in the power system may be caused, which can be regarded as a large power-flow disturbance brought by the system splitting. In general, such a splitting strategy is 


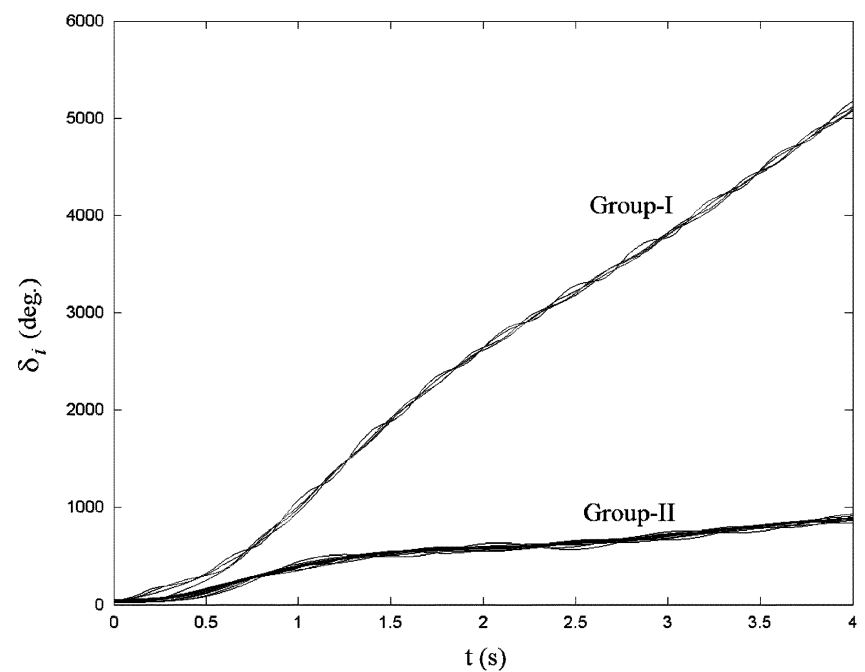

(a)

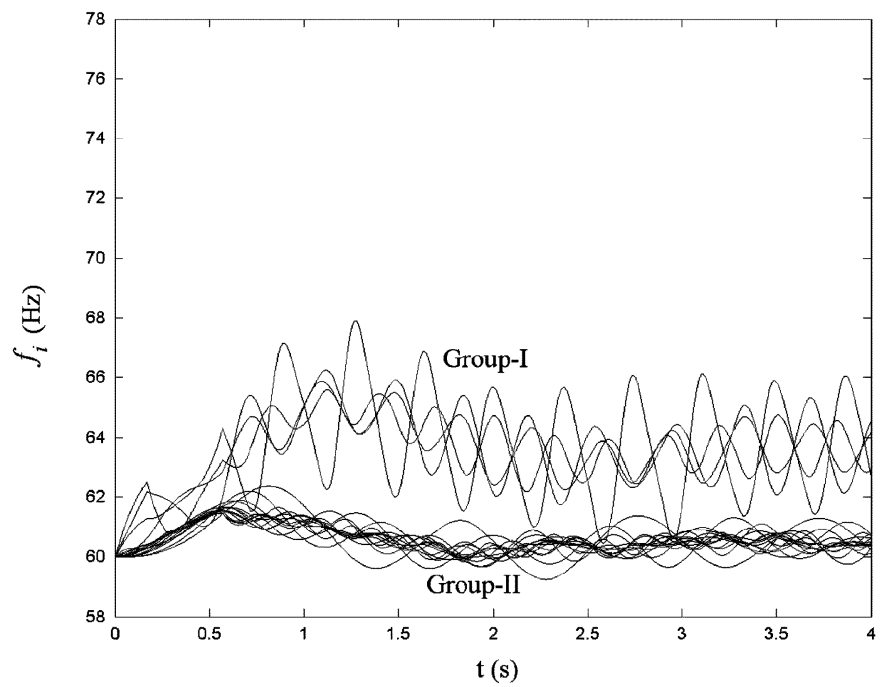

(b)

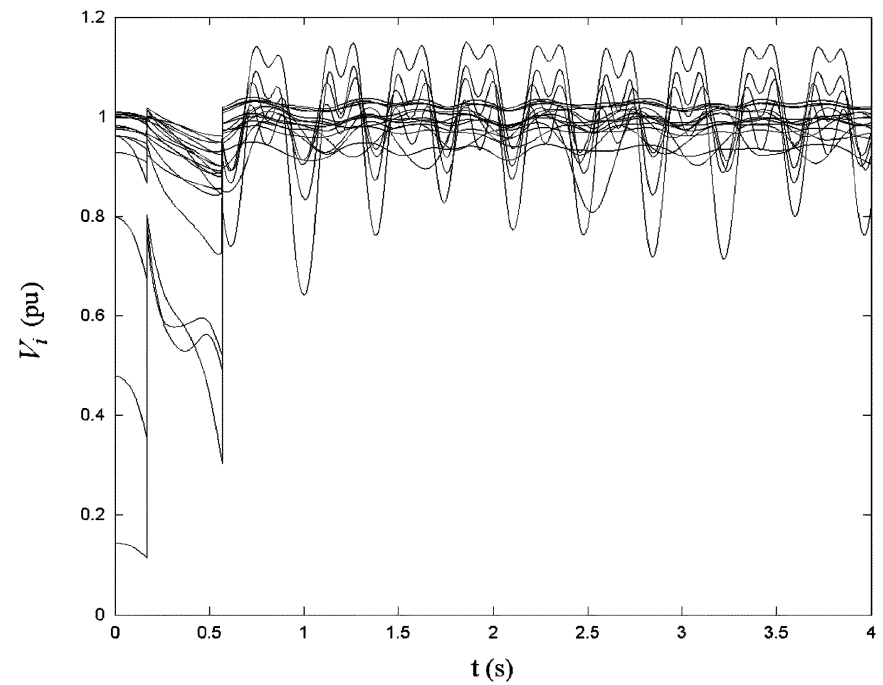

(c)

Fig. 6. Some dynamic curves of generators (without generator 31) after a successful system splitting. (a) Angular rotor swings of generators. (b) Frequencies of generators. (c) Voltages of generator buses.

very likely to produce unstable islands. Thus, a new constraint is introduced to exclude such a splitting strategy. Firstly, for a splitting strategy $S$, its two parameters are defined in (7) and (8) to reflect the degree of the power-flow disturbance brought by $S$

$$
\begin{aligned}
\gamma_{\text {Net }}(S) & =\sum_{e_{i j} \in S}\left|P_{i j}\right| / \sum_{P_{k}>0, v_{k} \in V} P_{k} \\
\gamma_{\text {Island }}(S) & =\max _{l=1, \ldots, m}\left(\sum_{\substack{e_{i j} \in S, v_{i}, v_{j} \in V^{l}}}\left|P_{i j}\right| / \sum_{P_{k}>0, v_{k} \in V^{l}} P_{k}\right) \text { (8) }
\end{aligned}
$$

where $P_{k}$ and $P_{i j}$ are respectively the real power of bus $k$ and the transmission real power of line $i-j$ before faults occur, $m$ is the number of the islands that $S$ produces, and $V^{1} \sim V^{m}$ are, respectively, the node sets of the $m$ islands. $P_{k}$ and $P_{i j}$ have been calculated in Period Layer from Short-term Load Forecasting data. Secondly, respectively set two threshold values $\Gamma_{\text {Net }}$ and $\Gamma_{\text {Island }}$ for $\gamma_{\text {Net }}(S)$ and $\gamma_{\text {Island }}(S)$. Then, the following constraint, TVC, can exclude the splitting strategies that bring large power-flow disturbances

$$
\left\{\begin{array}{l}
\gamma_{\text {Net }}(S) \leq \Gamma_{\text {Net }} \\
\gamma_{\text {Island }}(S) \leq \Gamma_{\text {Island }}
\end{array}\right.
$$

For determined $\Gamma_{\text {Net }}$ and $\Gamma_{\text {Island }}$, the proper splitting strategies that satisfy TVC are called "threshold value strategies", which, in fact, satisfy SSC, PBC, RLC, and TVC. In the rest of this subsection, the relation between threshold value strategies and feasible splitting strategies will be discussed.

Randomly select $N$ proper splitting strategies found by the OBDD method. Among the $N$ proper splitting strategies, use $N_{T}$ to denote the number of the threshold value strategies and use $N_{F}$ to denote the number of feasible splitting strategies. Obviously, $N_{T}$ can be regarded as a function of $\Gamma_{\text {Net }}$ and $\Gamma_{\text {Island. }}$. If $N_{T}>0$, define $R_{T}$ as

$$
R_{T}=1-N_{F} / N_{T}\left(N_{T}>0\right) .
$$

In fact, $R_{T}$ denotes the rate of the unfeasible splitting strategies among the $N_{T}$ threshold value strategies. The characteristics of $N_{T}\left(\Gamma_{\text {Net }}, \Gamma_{\text {Island }}\right)$ and $R_{T}\left(\Gamma_{\text {Net }}, \Gamma_{\text {Island }}\right)$ are helpful for analyzing the relation between threshold value strategies and feasible splitting strategies. For the 500 proper splitting strategies mentioned above, after their $\gamma_{\mathrm{Net}} \mathrm{S}$ and $\gamma_{\text {Island } \mathrm{S}}$ are calculated, the plots of $N_{T}\left(\Gamma_{\text {Net }}, \Gamma_{\text {Island }}\right)\left(\Gamma_{\text {Net }}, \Gamma_{\text {Island }} \in[0,0.5]\right)$ and $R_{T}\left(\Gamma_{\text {Net }}, \Gamma_{\text {Island }}\right)\left(\Gamma_{\text {Net }}, \Gamma_{\text {Island }} \in[0,0.5]\right.$ and $\left.N_{T}>0\right)$ can easily be obtained as shown in Fig. 7. From calculation results and Fig. 7, the follow three points can be found.

1) Larger $\Gamma_{\text {Net }}$ and $\Gamma_{\text {Island lead to larger }} N_{T}$, and usually lead to larger $R_{T}$, too.

2) If $\Gamma_{\text {Net }}<0.205$ or $\Gamma_{\text {Island }}<0.025$, then $N_{T}=0$, which means that no threshold value strategy exists.

3) There is an area in the " $\Gamma_{\text {Net }}-\Gamma_{\text {Island" plane. If point }}$ ( $\Gamma_{\text {Net }}, \Gamma_{\text {Island }}$ ) is in the area, then $N_{T}>0$ and $R_{T}=0$, which means that all threshold value strategies are feasible splitting strategies. 


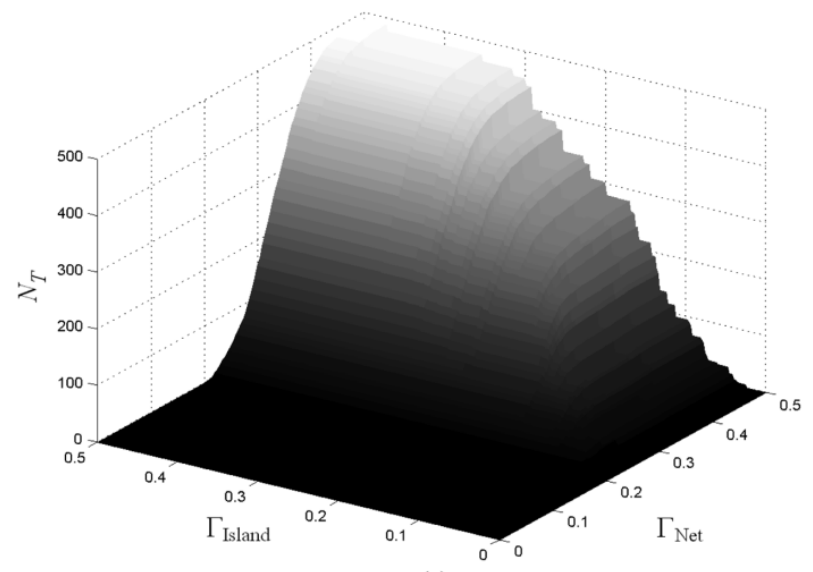

(a)

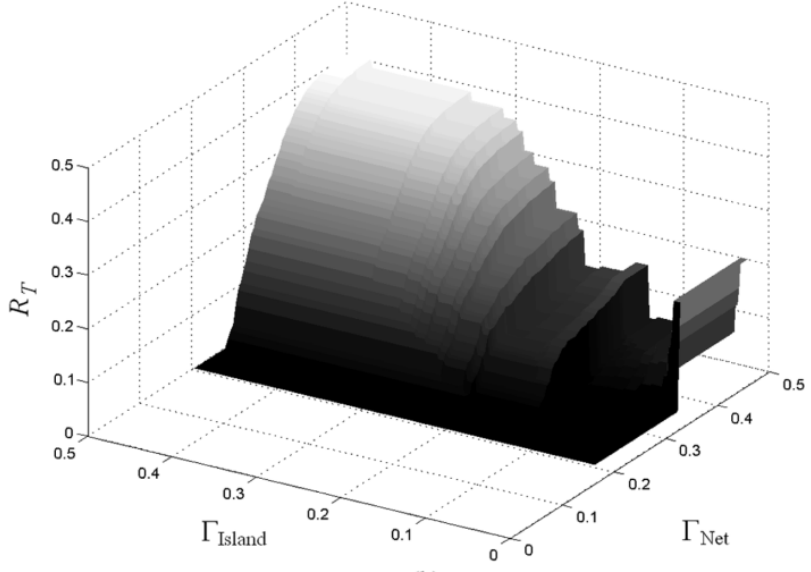

(b)

Fig. 7. Characteristics of $N_{T}$ and $R_{T}$ for 500 proper splitting strategies. (a) $N_{T}\left(\Gamma_{\text {Net }}, \Gamma_{\text {Island }}\right)$. (b) $N_{T}\left(\Gamma_{\text {Net }}, \Gamma_{\text {Island }}\right)\left(N_{T}>0\right)$.

From the above three points and the plots of $N_{T}\left(\Gamma_{\text {Net }}, \Gamma_{\text {Island }}\right)$ and $R_{T}\left(\Gamma_{\text {Net }}, \Gamma_{\text {Island }}\right)$, the " $\Gamma_{\text {Net }}-\Gamma_{\text {Island }}$ " plane can be partitioned into areas according to different $N_{T}$ and $R_{T}$. If given numbers $1=\alpha_{0}>\alpha_{1}>\alpha_{2}>\cdots>\alpha_{M}=0$, the " $\Gamma_{\text {Net }}-\Gamma_{\text {Island" }}$ plane can be partitioned into $M+2$ areas, denoted by $A(-1), A\left(\alpha_{0}\right), A\left(\alpha_{1}\right), \ldots$, and $A\left(\alpha_{M}\right)$, where

$$
\begin{aligned}
A(-1)= & \left\{\left(\Gamma_{\text {Net }}, \Gamma_{\text {Island }}\right) \mid N_{T}=0\right\} \\
A\left(\alpha_{0}\right)= & A(1) \\
= & \left\{\left(\Gamma_{\text {Net }}, \Gamma_{\text {Island }}\right) \mid N_{T}>0 \quad \text { and } \quad R_{T}=0\right\} \\
A\left(\alpha_{i}\right)= & \left\{\left(\Gamma_{\text {Net }}, \Gamma_{\text {Island }}\right) \mid N_{T}>0\right. \text { and } \\
& \left.\alpha_{i} \leq 1-R_{T}<\alpha_{i-1}\right\} \quad(i=1 \sim M) .
\end{aligned}
$$

Obviously, if point $\left(\Gamma_{\text {Net }}, \Gamma_{\text {Island }}\right) \in A(-1)$, no threshold value strategy exists, or in other words, TVC cannot be satisfied. If point $\left(\Gamma_{\text {Net }}, \Gamma_{\text {Island }}\right) \in A(1)$, all threshold value strategies are feasible splitting strategies. If point $\left(\Gamma_{\text {Net }}, \Gamma_{\text {Island }}\right) \in A\left(\alpha_{i}\right)(i>0)$, among all threshold value strategies, the rate of feasible splitting strategies (i.e., $N_{F} / N_{T}$ ) belongs to interval $\left[\alpha_{i}, \alpha_{i-1}\right)$. For example, for the above 500 proper splitting strategies, there are

$$
\begin{aligned}
A(-1)= & \left\{\left(\Gamma_{\text {Net }}, \Gamma_{\text {Island }}\right) \mid \Gamma_{\text {Net }}<0.205\right. \text { or } \\
& \left.\Gamma_{\text {Island }}<0.025\right\}
\end{aligned}
$$

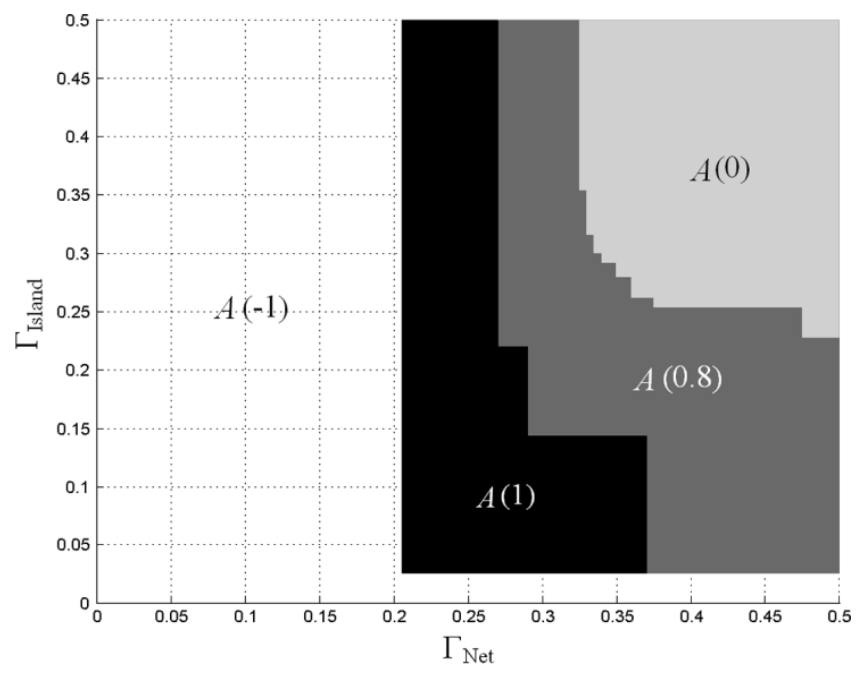

Fig. 8. Partition of the " $\Gamma_{\text {Net }}-\Gamma_{\text {Island" }}$ plane.

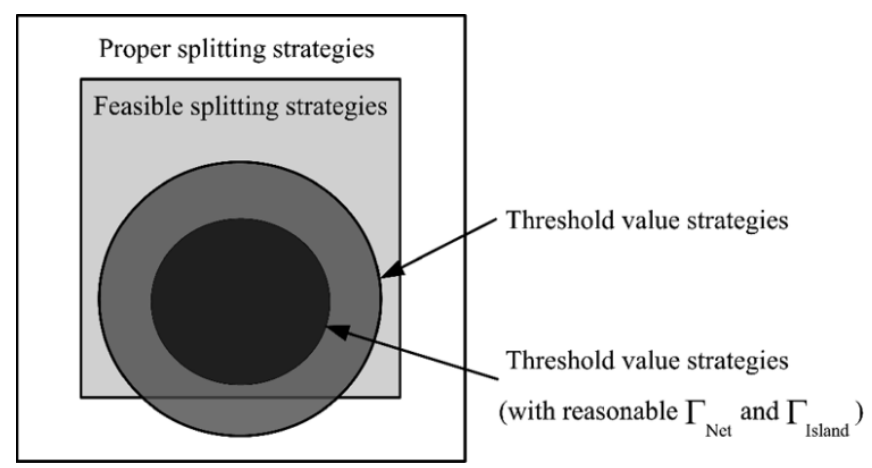

Fig. 9. Relations between proper splitting strategies, feasible splitting strategies and threshold value strategies.

and

$$
\begin{aligned}
A(1) \doteq & \left\{\left(\Gamma_{\text {Net }}, \Gamma_{\text {Island }}\right) \mid{ }^{\prime} \Gamma_{\text {Net }} \in[0.205,0.365]\right. \text { and } \\
& \Gamma_{\text {Island }} \in[0.025,0.142] " \\
& \text { or }{ }^{\prime} \Gamma_{\text {Net }} \in[0.205,0.289] \text { and } \\
& \Gamma_{\text {Island }} \in[0.142,0.218] " \\
& \text { or }{ }^{\prime} \Gamma_{\text {Net }} \in[0.205,0.268] \text { and } \\
& \left.\Gamma_{\text {Island }} \in[0.218,0.5] "\right\} .
\end{aligned}
$$

A partition of the " $\Gamma_{\text {Net }}-\Gamma_{\text {Island" plane with } M=2, \alpha_{1}=}$ 0.8 , and $\alpha_{2}=0$ is shown in Fig. 8. For arbitrary $\Gamma_{\text {Net }}$ and $\Gamma_{\text {Island }}$ selected in the area $A(0.8)$, more than $80 \%$ threshold value strategies are feasible splitting strategies. Moreover, transient simulation results show that, if $\left(\Gamma_{\text {Net }}, \Gamma_{\text {Island }}\right) \in A(1)$, the ASDP's of all threshold value strategies (also, feasible splitting strategies) are longer than $0.39 \mathrm{~s}$. That means that if $\Gamma_{\mathrm{Net}}$ and $\Gamma_{\text {Island }}$ are selected in $A(1)$, TVC can exclude all unfeasible splitting strategies and the feasible splitting strategies with comparatively short ASDPs.

From the above results, the relations between proper splitting strategies, feasible splitting strategies and threshold value strategies are depicted in Fig. 9, where the white rectangle 
represents the set of all proper splitting strategies, the gray rectangle represents the set of all feasible splitting strategies, and the two circles represent two sets of threshold value strategies with different threshold values. As is depicted by the bigger circle, threshold value strategies perhaps contain some unfeasible splitting strategies. However, if $\Gamma_{\text {Net }}$ and $\Gamma_{\text {Island }}$ are reasonably selected, all threshold value strategies are feasible splitting strategies as is depicted by the smaller circle. Therefore, it is very important to select reasonable threshold values $\Gamma_{\text {Net }}$ and $\Gamma_{\text {Island }}$ for TVC.

The following approach is proposed to offline select $\Gamma_{\text {Net }}$ and $\Gamma_{\text {Island }}$ for a power system.

1) For some typical cases in which loss of synchronism happens, respectively find enough proper splitting strategies by the OBDD method to compose a set of proper splitting strategies.

2) For this set of proper splitting strategies, draw the plots of $N_{T}\left(\Gamma_{\text {Net }}, \Gamma_{\text {Island }}\right)$ and $R_{T}\left(\Gamma_{\text {Net }}, \Gamma_{\text {Island }}\right)$ by means of checking TVC with different threshold values and performing power system transient simulations on computers.

3) Select a positive number $\beta$ close to 0 (e.g., $\beta=0.05$ ) and define area $B$ as (14)

$$
B=\left\{\left(\Gamma_{\text {Net }}, \Gamma_{\text {Island }}\right) \mid N_{T}>0 \quad \text { and } \quad R_{T} \leq \beta\right\}
$$

4) Select a point $\left(\Gamma_{\text {Net }, 0}, \Gamma_{\text {Island, } 0}\right)$ in the central part of area $B$. Let $\Gamma_{\text {Net }}=\Gamma_{\text {Net }, 0}$ and $\Gamma_{\text {Island }}=\Gamma_{\text {Island }, 0}$.

Remark: This approach is based on the following consideration. For determined power system and $\alpha_{1} \sim \alpha_{M}$, areas $A(-1), A\left(\alpha_{0}\right), A\left(\alpha_{1}\right), \ldots$, and $A\left(\alpha_{M}\right)$ are not completely fixed because the locations and types of faults are not fixed, and the proper splitting strategies that are used to partition the " $\Gamma_{\mathrm{Net}}-\Gamma_{\text {Island }}$ " plane are not fixed, either. However, the $\gamma_{\mathrm{Net}} \mathrm{S}$ and $\gamma_{\text {Island }} \mathrm{S}$ of feasible splitting strategies usually have some common characteristics, so they may be more concentrated in a region of the " $\Gamma_{\text {Net }}-\Gamma_{\text {Island" plane. This region is very }}$ possible to cover part or even the whole $A(1)$. The above area $B$ is just an estimate of this region. Therefore, the TVC with the $\Gamma_{\text {Net, } 0}$ and $\Gamma_{\text {Island, } 0}$ can easily find feasible splitting strategies.

Furthermore, the relations between $\left(\Gamma_{\text {Net }, 0}, \Gamma_{\text {Island, } 0}\right)$ and real $A(-1), A(1), A\left(\alpha_{1}\right), \ldots$, and $A\left(\alpha_{M}\right)$ are analyzed as follows. There must be three possibilities.

1) If point $\left(\Gamma_{\text {Net }, 0}, \Gamma_{\text {Island, } 0}\right) \in A(1)$, all threshold value strategies are feasible splitting strategies.

2) If point $\left(\Gamma_{\text {Net }, 0}, \Gamma_{\text {Island, } 0}\right) \in A\left(\alpha_{i}\right)\left(\alpha_{i}\right.$ is usually a number close to 1$)$, a threshold value strategy still has a high probability $\left(\geq \alpha_{i}\right)$ to be a feasible splitting strategy. Even if it is an unfeasible splitting strategy, only few islands may be unstable after it splits the power system into islands. With the aid of effective emergency control actions (e.g., generator tripping and load shedding), all islands can easily be stabilized to lead to a successful system splitting.

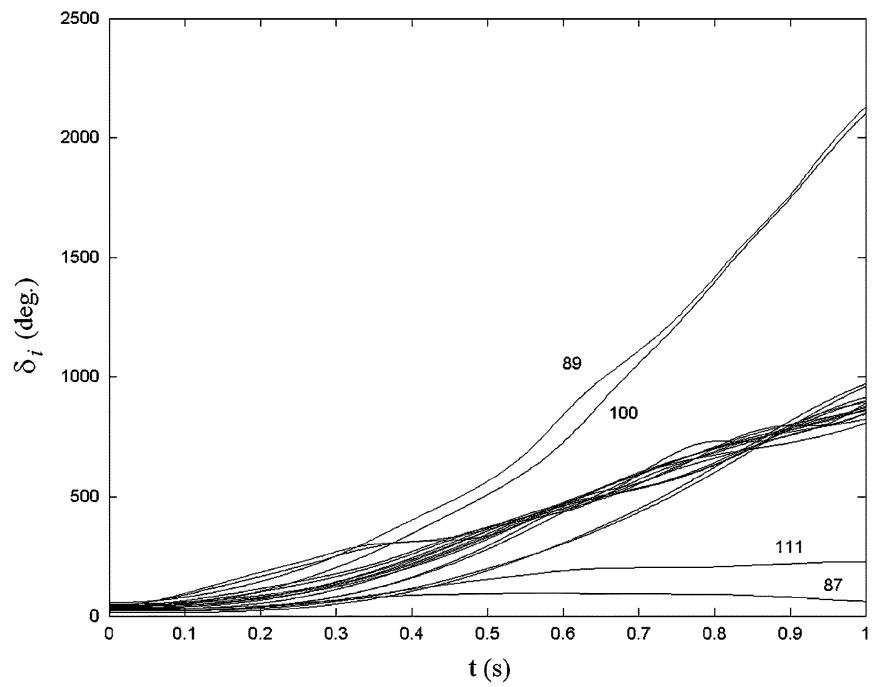

(a)

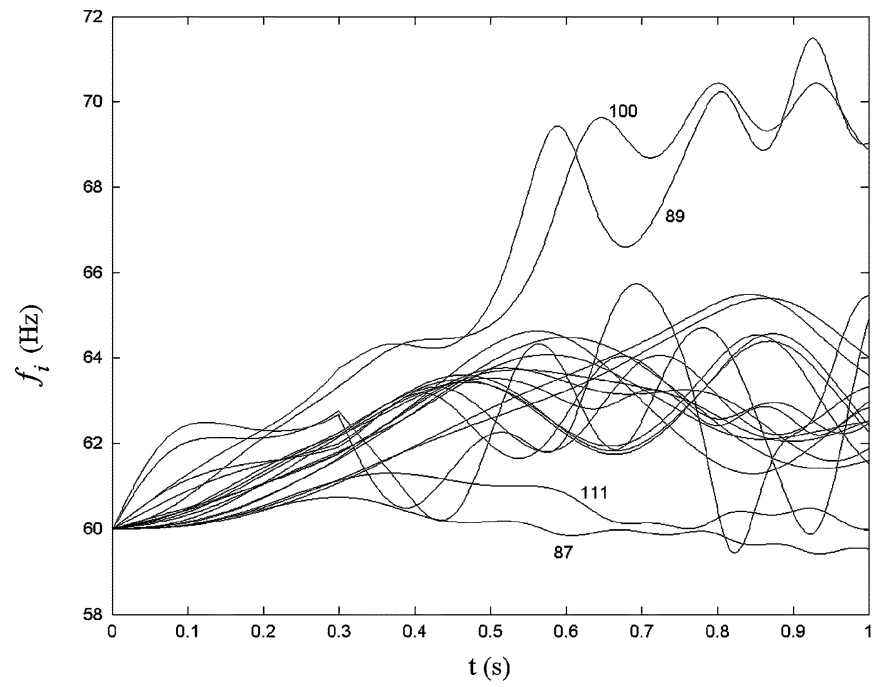

(b)

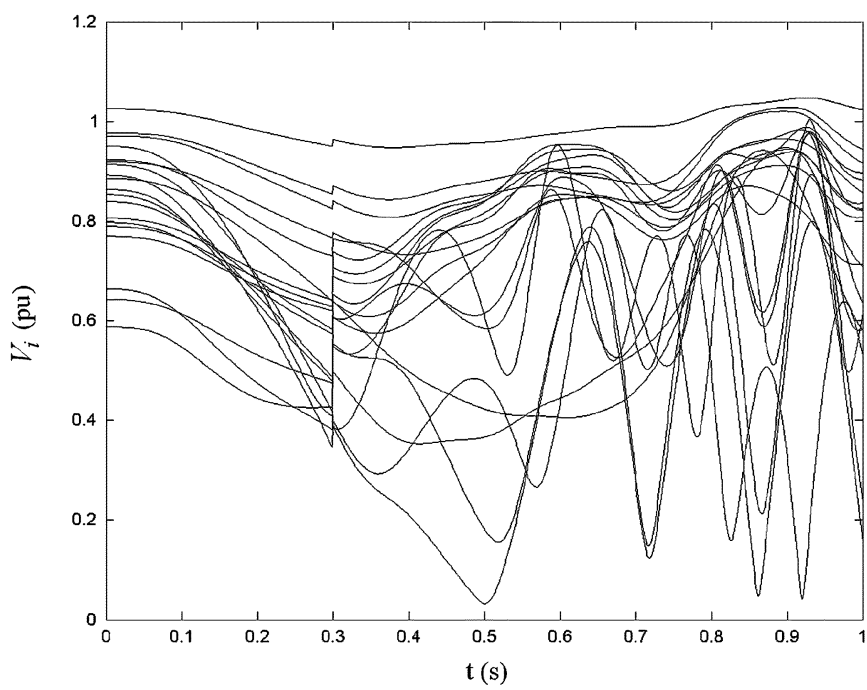

(c)

Fig. 10. Some dynamic curves of generators after a fault is cleared at line 68-81. (a) Angular rotor swings of all generators. (b) Frequencies of all generators. (c) Voltages of all generator buses.

3) If point $\left(\Gamma_{\text {Net }, 0}, \Gamma_{\text {Island, } 0}\right) \in A(-1)$, no threshold value strategy can be found. For each proper splitting strategy 
TABLE III

Simulations Results ABout 168 Faults

\begin{tabular}{|c|c|c|c|c|c|c|}
\hline No. & $\begin{array}{c}\text { Fault } \\
\text { locations }\end{array}$ & $P_{i j}(\mathrm{MW})$ & Three-phase faults & $\begin{array}{l}\text { Double-line-to-ground } \\
\text { faults }\end{array}$ & Line-to-line faults & $\begin{array}{l}\text { Single-line-to- } \\
\text { ground faults }\end{array}$ \\
\hline $1 \mathrm{a}$ & $4^{\#}-5$ & \multirow{2}{*}{103.3} & 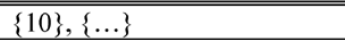 & 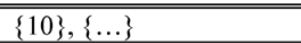 & 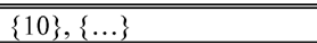 & 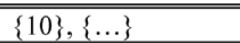 \\
\hline $1 \mathrm{~b}$ & $4-5^{\#}$ & & $\{10\},\{\ldots\}$ & $\{10\},\{\ldots\}$ & $\{10\},\{\ldots\}$ & $\{10\},\{\ldots\}$ \\
\hline $2 a$ & $15^{\#}-17$ & \multirow{2}{*}{103.8} & $\{10\},\{25,26\},\{\ldots\}$ & $\{10\},\{25,26\},\{\ldots\}$ & $\{10\},\{25,26\},\{\ldots\}$ & $\{10\},\{\ldots\}$ \\
\hline $2 \mathbf{b}$ & $15-17^{\#}$ & & $\{10\},\{25,26\},\{\ldots\}$ & $\{10\},\{25,26\},\{\ldots\}$ & $\{10\},\{25,26\},\{\ldots\}$ & $\{10\},\{25,26\},\{\ldots\}$ \\
\hline $3 a$ & $25^{\#}-27$ & \multirow{2}{*}{143.5} & $\{10\},\{25,26\},\{\ldots\}$ & STABLE & STABLE & STABLE \\
\hline $3 b$ & $25-27^{\#}$ & & STABLE & STABLE & STABLE & STABLE \\
\hline $4 a$ & $26^{\#}-30$ & \multirow{2}{*}{223.7} & $\{10\},\{25\},\{26\},\{\ldots\}$ & STABLE & $\{25,26\},\{\ldots\}$ & STABLE \\
\hline $4 \mathrm{~b}$ & $26-30^{\#}$ & & $\{10\},\{25,26\},\{\ldots\}$ & $\{10\},\{25,26\},\{\ldots\}$ & $\{10\},\{25,26\},\{\ldots\}$ & $\{10\},\{25,26\},\{\ldots\}$ \\
\hline $5 \mathrm{a}$ & $42^{\#}-49$ & \multirow{2}{*}{129.7} & STABLE & STABLE & STABLE & STABLE \\
\hline $5 \mathrm{~b}$ & $42-49^{\#}$ & & STABLE & STABLE & STABLE & STABLE \\
\hline $6 a$ & $60^{\#-61}$ & \multirow{2}{*}{112.2} & STABLE & STABLE & STABLE & STABLE \\
\hline $6 \mathrm{~b}$ & $60-61^{\#}$ & & $\{61\},\{\ldots\}$ & STABLE & STABLE & STABLE \\
\hline $7 \mathrm{a}$ & $63^{\#}-64$ & \multirow{2}{*}{151.6} & STABLE & STABLE & STABLE & STABLE \\
\hline $7 b$ & $63-64^{\#}$ & & STABLE & STABLE & STABLE & STABLE \\
\hline $8 \mathrm{a}$ & $38^{\#-65}$ & \multirow{2}{*}{181.3} & $\{10\},\{25,26\},\{\ldots\}$ & STABLE & $\{10,12,25,26\},\{31\},\{\ldots\}$ & STABLE \\
\hline $8 \mathrm{~b}$ & $38-65^{\#}$ & & $\{87\},\{\ldots\}$ & STABLE & STABLE & STABLE \\
\hline $9 \mathrm{a}$ & $64^{\#}-65$ & \multirow{2}{*}{182.9} & STABLE & STABLE & STABLE & STABLE \\
\hline $9 \mathrm{~b}$ & $64-65^{\#}$ & & $\{65\},\{31,46,87,111\},\{\ldots\}$ & STABLE & STABLE & STABLE \\
\hline $10 \mathrm{a}$ & $49^{\#}-66$ & \multirow{2}{*}{264.4} & $\{49\},\{\ldots\}$ & STABLE & STABLE & STABLE \\
\hline $10 \mathrm{~b}$ & $49-66^{\#}$ & & $\{66\},\{\ldots\}$ & STABLE & STABLE & STABLE \\
\hline $11 \mathrm{a}$ & $69^{\#-70}$ & \multirow{2}{*}{108.4} & $\{69\},\{\ldots\}$ & STABLE & STABLE & STABLE \\
\hline $11 \mathrm{~b}$ & $69-70^{\#}$ & & STABLE & STABLE & STABLE & STABLE \\
\hline $12 a$ & $69^{\#-75}$ & \multirow{2}{*}{110.1} & $\{69\},\{\ldots\}$ & STABLE & STABLE & STABLE \\
\hline $12 b$ & $69-75^{\#}$ & & STABLE & STABLE & STABLE & STABLE \\
\hline $13 \mathrm{a}$ & $77^{\#}-80$ & \multirow{2}{*}{141.0} & STABLE & STABLE & STABLE & STABLE \\
\hline $13 b$ & $77-80^{\#}$ & & $\{80\},\{89\},\{\ldots\}$ & STABLE & STABLE & \\
\hline $14 a$ & $89^{\#}-90$ & \multirow{2}{*}{169} & $\{89\},\{\ldots\}$ & STABLE & $\{89\},\{\ldots\}$ & STABLE \\
\hline $14 \mathrm{~b}$ & $89-90^{\#}$ & & $\{89\},\{\ldots\}$ & $\{89\},\{\ldots\}$ & $\{89\},\{\ldots\}$ & STABLE \\
\hline $15 a$ & $89^{\#-92}$ & \multirow{2}{*}{265.2} & $\{89\},\{\ldots\}$ & STABLE & $\{89\},\{\ldots\}$ & STABLE \\
\hline $15 b$ & $89-92^{\#}$ & & $\{89\},\{\ldots\}$ & $\{89\},\{\ldots\}$ & $\{89\},\{\ldots\}$ & $\{89\},\{\ldots\}$ \\
\hline $16 \mathrm{a}$ & $100^{\#}-103$ & \multirow{2}{*}{121.8} & $\{89\},\{100\},\{\ldots\}$ & STABLE & STABLE & STABLE \\
\hline $16 \mathrm{~b}$ & $100-103^{\#}$ & & STABLE & STABLE & STABLE & STABLE \\
\hline $17 \mathrm{a}$ & $68^{\#}-81$ & \multirow[t]{2}{*}{43.9} & $\begin{array}{l}\{69\},\{80,89,100\},\{87\} \\
\{111,103\},\{\ldots\}\end{array}$ & $\begin{array}{l}\{69\},\{80,89,100\},\{87\}, \\
\{103,111\},\{\ldots\}\end{array}$ & $\begin{array}{l}\{69\},\{80,89,100\},\{87\}, \\
\{103,111\},\{\ldots\}\end{array}$ & STABLE \\
\hline $17 \mathrm{~b}$ & $68-81^{\#}$ & & $\{89,100\},\{87\},\{111\},\{\ldots\}$ & STABLE & STABLE & STABLE \\
\hline $18 \mathrm{a}$ & $49^{\#}-69$ & \multirow{2}{*}{46.5} & STABLE & STABLE & STABLE & STABLE \\
\hline $18 \mathrm{~b}$ & $49-69^{\#}$ & & STABLE & STABLE & STABLE & STABLE \\
\hline $19 \mathrm{a}$ & $49^{\#}-54$ & \multirow{2}{*}{51.7} & STABLE & STABLE & STABLE & STABLE \\
\hline $19 b$ & $49-54^{\#}$ & & STABLE & STABLE & STABLE & STABLE \\
\hline $20 \mathrm{a}$ & $59^{\#}-61$ & \multirow{2}{*}{30.4} & STABLE & STABLE & STABLE & STABLE \\
\hline $20 \mathrm{~b}$ & $59-61^{\#}$ & & STABLE & STABLE & STABLE & STABLE \\
\hline $21 \mathrm{a}$ & $54^{\#}-59$ & \multirow{2}{*}{46.5} & STABLE & STABLE & STABLE & STABLE \\
\hline $21 \mathrm{~b}$ & $54-59^{\#}$ & & STABLE & STABLE & STABLE & STABLE \\
\hline
\end{tabular}

$S$, define $d_{\gamma}(S)$ as (15). Then, select the proper splitting strategy that makes $d_{\gamma}$ smallest

$$
d_{\gamma}(S)=\sqrt{\left(\gamma_{\text {Net }}(S)-\Gamma_{\text {Net }, 0}\right)^{2}+\left(\gamma_{\text {Island }}(S)-\Gamma_{\text {Island }, 0}\right)^{2}} .
$$

In fact, $d_{\gamma}(S)$ is the distance between the two points $\left(\gamma_{\text {Net }}(S), \gamma_{\text {Island }}(S)\right)$ and $\left(\Gamma_{\text {Net }, 0}, \Gamma_{\text {Island }, 0}\right)$ in the " $\Gamma_{\text {Net }}-\Gamma_{\text {Island" plane. The selected proper splitting }}$ strategy is called "shortest-distance splitting strategy" and denoted by $S_{d}$. Obviously, point $\left(\gamma_{\text {Net }}\left(S_{d}\right), \gamma_{\text {Island }}\left(S_{d}\right)\right)$ may be in $A(1)$ or $A\left(\alpha_{i}\right)\left(\alpha_{i} \approx 1\right)$. For the former situation, $S_{d}$ itself is feasible splitting strategy; the latter situation is the same as 2) above.

If it is true that no threshold value strategy is found, the shortest-distance splitting strategy can be found within ex- tremely short time since the $\gamma_{\mathrm{Net}}$ and $\gamma_{\mathrm{Island}}$ of each proper splitting strategy has been calculated in the checking of TVC. The above discussion shows that if $\Gamma_{\text {Net }}$ and $\Gamma_{\text {Island }}$ are selected by the above approach, a proper splitting strategy that has a high possibility to be a feasible splitting strategy can be given.

\section{Verifying the Function of Threshold Value Constraint in Determining Feasible Splitting Strategies}

Here, threshold values $\Gamma_{\text {Net }}$ and $\Gamma_{\text {Island }}$ are firstly selected offline by the approach proposed in last subsection. Then, many case studies on the IEEE 118-bus system demonstrate that TVC is able to find feasible splitting strategies.

Consider the 500 proper splitting strategies mentioned above and let $\beta=0$. Thus, area $B$ is just the " $A(1)$ " in Fig. 8. Select $\Gamma_{\text {Net }, 0}=0.3$ and $\Gamma_{\text {Island }, 0}=0.1$ in the central part of $B$. 
Firstly, randomly select another 500 proper splitting strategies for the case discussed in Section IV-B. After TVC is checked for the 500 strategies, 90 threshold value strategies are found. Then, by means of power system transient simulations, it is found that the 90 strategies are all feasible splitting strategies and their ASDPs $>0.39 \mathrm{~s}$. The results imply that, for this case, the TVC with $\Gamma_{\text {Net, } 0}$ and $\Gamma_{\text {Island, } 0}$ excludes all unfeasible splitting strategies and the feasible splitting strategies with ASDPs $\leq 0.39$.

Secondly, as shown in Table III, another 21 critical transmission lines are selected. The transmission real powers of the former 16 lines are all larger than $100 \mathrm{MW}$ before faults occur. The two terminal buses of each of the latter five lines are both near generators. Hence, the faults occurring on the 21 lines easily lead to loss of synchronism of generators. Respectively set four types of faults at each bus of the 21 lines: a three-phase fault, a line-to-line fault, a double-line-to-ground fault and a single-line-to-ground fault. Thus, $21 \times 2 \times 4=168$ faults are considered. The location of each fault is indicated by "\#" in Table III. Assume that all the faults occur at the time $t=0.0 \mathrm{~s}$ and are cleared after $0.3 \mathrm{~s}$, and no other emergency control actions are applied. Then perform power system transient simulation for each of the 168 faults and list the simulation results in Table III. If a fault leads to loss of synchronism of generators, asynchronous groups of generators are given in the corresponding cell of the table, where " $\{\ldots\}$ " means "the other generators"; otherwise, a "STABLE" is placed. As an example, for the three-phase fault at $68-81^{\#}$ (no. 17b), some dynamic curves of all generators are shown in Fig. 10. From Table III, it can be found that the faults at 23 locations lead to loss of synchronism, and three-phase faults generally cause more serious effects on the stability of the system than the other three types of faults. Moreover, it can be observed that the fault at $38-65^{\#}$ (no. 8b) only makes "unimportant" generator 87 become asynchronous with the others. Simulations show that after generator 87 is tripped, the rest of the system becomes stable. Therefore, only the three-phase faults at the other 22 locations (indicated by gray cells and bold letters in the first column of Table III) are studied. For each of the 22 faults, enough proper splitting strategies can be found by the OBDD method. Randomly select 500 proper splitting strategies for each fault, and check TVC for the strategies. If threshold value strategies exist, transient simulations can find whether they are feasible splitting strategies. The final results are follows.

For the ten faults given in Table IV, threshold value strategies exit. From Table IV, almost all threshold value strategies are feasible splitting strategies. In detail, for six faults, $100 \%$ threshold value strategies are feasible splitting strategies, and for 4 faults, respectively $96.2 \%, 91.7 \%, 86.7 \%$, and 95.7\% threshold value strategies are feasible splitting strategies. Furthermore, it can be found that the ASDPs of most threshold value strategies are longer than $0.5 \mathrm{~s}$. As an example, for the three-phase fault at $68-81^{\#}$ (no. 17b), Fig. 11 gives some dynamic curves of generators after a feasible splitting strategy is applied with $\mathrm{SDP}=0.6 \mathrm{~s}$. The feasible splitting strategy trips "unimportant" generators 87,103 , and 111. It can be observed that the rotor angles, frequencies and bus voltages of the other generators are all stable, and hence two stable islands are produced.

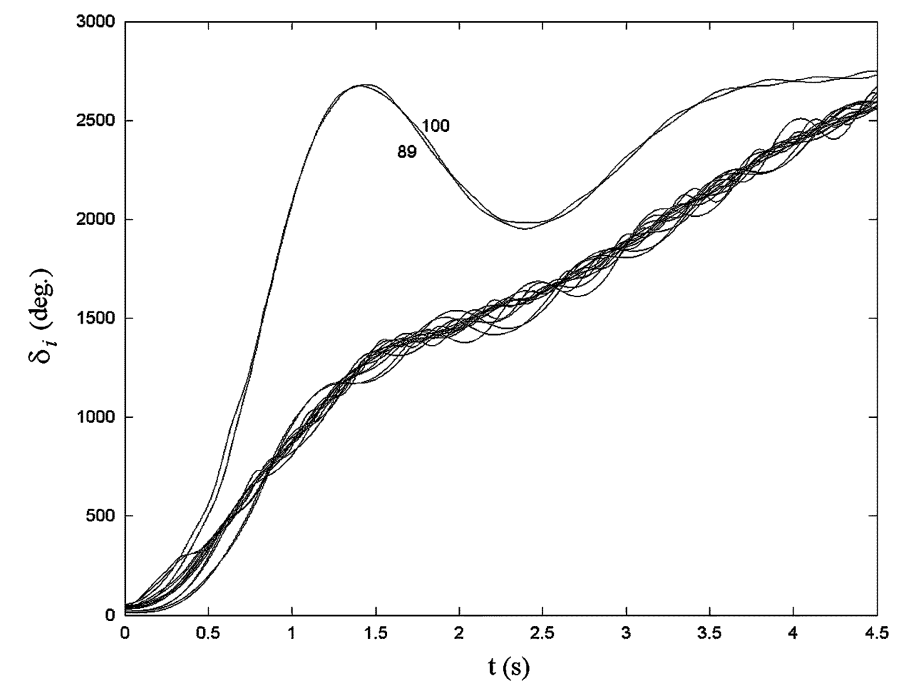

(a)

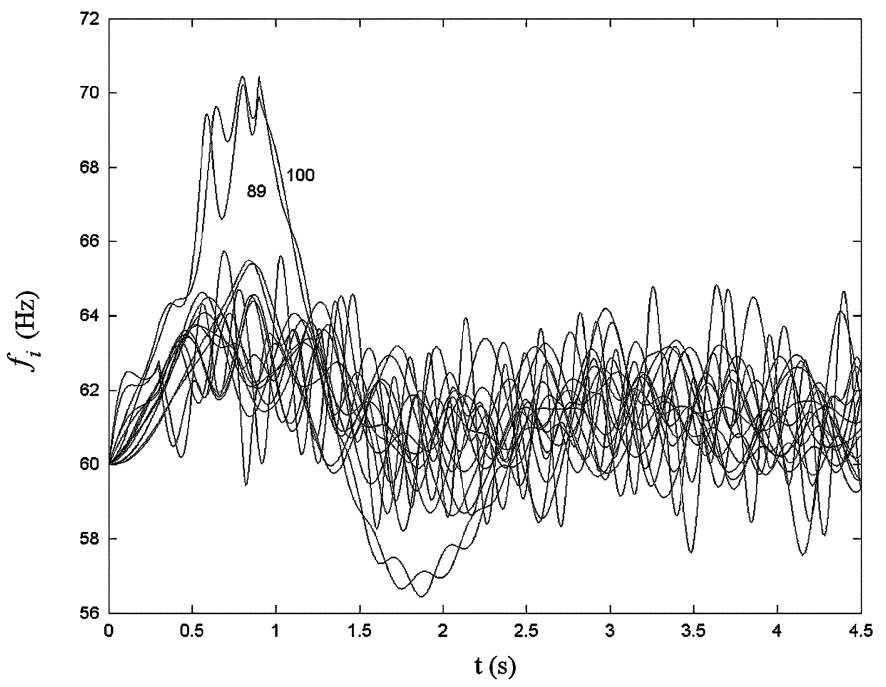

(b)

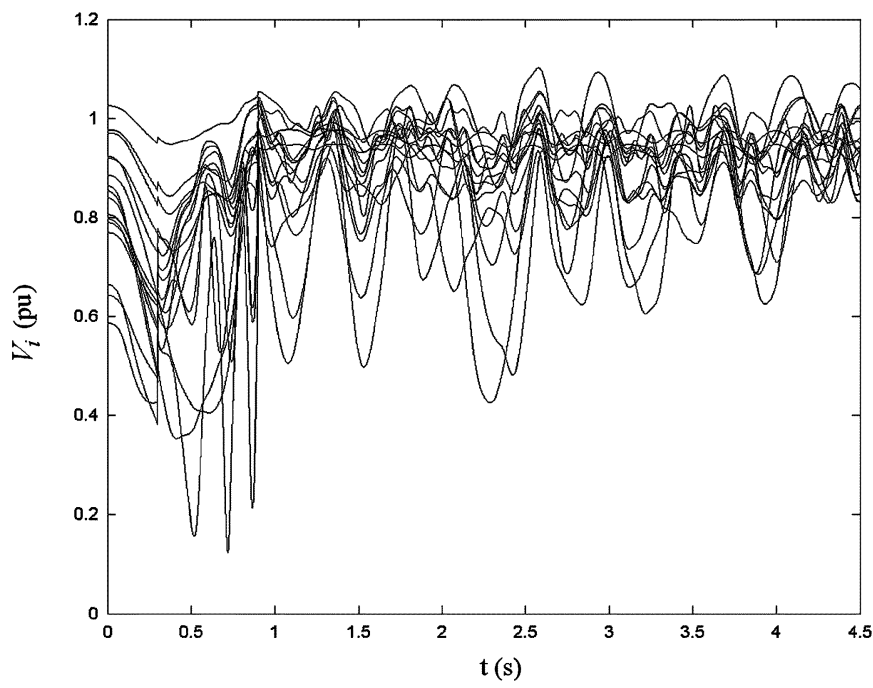

(c)

Fig. 11. Some dynamic curves of generators (without generators 87, 103, and 111) after a successful system splitting. (a) Angular rotor swings of generators. (b) Frequencies of generators. (c) Voltages of generator buses.

For the other 12 faults, namely the three-phase faults at $4^{\#}-5$ (no. $1 \mathrm{a}$ ), $4-5^{\#}$ (no. $1 \mathrm{~b}$ ), $15^{\#}-17$ (no. $2 \mathrm{a}$ ), $15-17^{\#}$ (no. $2 \mathrm{~b}$ ), 
TABLE IV

FEASIBILITY OF THE THRESHOLD VALUE STRATEGIES FOR TEN FAULTS

\begin{tabular}{|c|c|c|c|c|c|c|}
\hline \multirow{2}{*}{ No. } & \multirow{2}{*}{$\begin{array}{c}\text { Fault } \\
\text { locations }\end{array}$} & \multirow{2}{*}{$\begin{array}{l}\text { The number of threshold } \\
\text { value strategies }\left(=N_{T}\right)\end{array}$} & \multicolumn{2}{|c|}{ Feasible splitting strategies } & \multicolumn{2}{|c|}{ Feasible splitting strategies with $A S D P \mathrm{~s}>0.5 \mathrm{~s}$} \\
\hline & & & Number $\left(=N_{F}\right)$ & Percentage $\left(=N_{F} / N_{T}\right)$ & Number & Percentage \\
\hline $4 \mathrm{~b}$ & $26-30^{\#}$ & 20 & 20 & $100 \%$ & 20 & $100 \%$ \\
\hline $8 \mathrm{a}$ & $38^{\#}-65$ & 32 & 32 & $100 \%$ & 32 & $100 \%$ \\
\hline $9 \mathrm{~b}$ & $64-65^{\#}$ & 106 & 102 & $96.2 \%$ & 77 & $72.6 \%$ \\
\hline $10 \mathrm{a}$ & $49^{\#}-66$ & 2 & 2 & $100 \%$ & 2 & $100 \%$ \\
\hline $10 \mathrm{~b}$ & $49-66^{\#}$ & 24 & 22 & $91.7 \%$ & 22 & $91.7 \%$ \\
\hline $11 \mathrm{a}$ & $69^{\#}-70$ & 7 & 7 & $100 \%$ & 7 & $100 \%$ \\
\hline $12 \mathrm{a}$ & $69^{\#}-75$ & 6 & 6 & $100 \%$ & 6 & $100 \%$ \\
\hline $13 \mathrm{~b}$ & $77-80^{\#}$ & 15 & 13 & $86.7 \%$ & 13 & $86.7 \%$ \\
\hline $17 \mathrm{a}$ & $68^{\#}-81$ & 23 & 22 & $95.7 \%$ & 22 & $95.7 \%$ \\
\hline $17 \mathrm{~b}$ & $68-81^{\#}$ & 8 & 8 & $100 \%$ & 8 & $100 \%$ \\
\hline
\end{tabular}

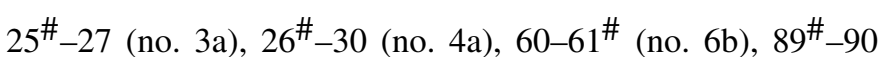
(no. $14 \mathrm{a}$ ), 89-90" (no. 14b), 89\#-92 (no. 15a), 89-92\# (no. $15 \mathrm{~b}$ ) and $100^{\#-103}$ (no. 16a), no threshold value strategy exists. Among the 500 proper splitting strategies for each of the 12 faults, select the shortest-distance splitting strategy. Transient simulations show that all the 12 shorter-distance splitting strategies are feasible splitting strategies.

In the above simulations, it takes less than $0.01 \mathrm{~s}$ to check TVC for one proper splitting strategy (by means of PC with a Pentium IV 1.4-GHz processor and 256-MB RAM).

To summarize, the above simulation results show the following.

1) Although the threshold values $\Gamma_{\text {Net }, 0}$ and $\Gamma_{\text {Island, } 0}$ are offline selected based on only 500 proper splitting strategies, they are fairly reasonable for about half of studied cases. For the cases, threshold value strategies exist and almost all $(86.7 \%-100 \%)$ threshold value strategies are feasible splitting strategies.

2) For the other studied cases, the threshold values $\Gamma_{\text {Net, } 0}$ and $\Gamma_{\text {Island, } 0}$ are too small to find threshold value strategies. However, their shortest-distance splitting strategies are all feasible splitting strategies.

3) Checking TVC for a proper splitting strategy can be completed within extremely short time, and a shortest-distance splitting strategy can quickly be found.

Finally, it can be concluded that, by means of TVC, feasible splitting strategies can effectively be found from the proper splitting strategies given by the OBDD method. Thus, if TVC is introduced into the OBDD method, and the OBDD method is properly modified, it is possible to online find a feasible splitting strategy by the modified method. Such a modified method for quickly generating feasible splitting strategies will be reported in our next paper.

\section{CONCLUSION}

This paper studies the feasibility of the proper splitting strategies given by the OBDD method. Simulation results show that among the proper splitting strategies, a considerable number of strategies are feasible splitting strategies, which can produce stable islands after they are applied. Moreover, TVC is proposed to exclude unfeasible splitting strategies. An approach is presented to offline select the threshold values for TVC. Further simulation results demonstrate that, if the threshold values of TVC are reasonably selected, feasible splitting strategies can effectively be found from proper splitting strategies to successfully split the power system into stable islands.

\section{REFERENCES}

[1] R. E. Bryant, "Graph-based algorithms for Boolean function manipulation," IEEE Trans. Comput., vol. C-35, no. 8, pp. 677-691, Aug. 1986.

[2] Q. Zhao et al., "A study of system splitting strategies for island operation of power system: A two-phase method based on OBDDs," IEEE Trans. Power Syst., vol. 18, no. 4, pp. 1556-1565, Nov. 2003.

[3] K. Sun, D. Zheng, and Q. Lu, "Splitting strategies for islanding operation of large-scale power systems using OBDD-based methods," IEEE Trans. Power Syst., vol. 18, no. 2, pp. 912-923, May 2003.

[4] A. A. Fouad and V. Vittal, Power System Transient Stability Analysis Using the Transient Energy Function Method. Englewood Cliffs, NJ: Prentice Hall, 1992.

[5] P. M. Anderson and A. A. Fouad, Power System Control and Stability (Revised Printing). New York: IEEE Press, 1994.

[6] [Online]. Available: http://www.ee.washington.edu/research/pstca/

[7] A. R. Bergen and V. Vittal, Power Systems Analysis. Englewood Cliffs, NJ: Prentice-Hall, 2000.

[8] J. Machowski, J. W. Bialek, and J. R. Bumby, Power System Dynamics and Stability. New York: Wiley, 1997.

Kai Sun received the B.S. degree in automation, the M.S. degree in control theory and control engineering, and the Ph.D. degree in control science and engineering from the Department of Automation at Tsinghua University, Beijing, China, in 1999, 2002, and 2004, respectively.

His research interests include power systems, discrete event dynamic systems, hybrid systems, and complex large systems.

Da-Zhong Zheng received the diploma in automatic control from Tsinghua University, Beijing, China, in 1959.

Since 1959, he has been with the Department of Automatic Control, Tsinghua University, where he is a Professor in control theory and engineering. He was a Visiting Scholar in Department of Electrical Engineering, State University of New York at Stony Brook, from 1981 to 1983 and in 1993. His research interests include linear systems, discrete event dynamic systems, and power systems. He has published many journal papers and five books.

Mr. Zheng is Vice Chairman of the Control Theory Technical Committee for Chinese Association of Automation (CAA) and a Deputy Editor-In-Chief of Acta Automatica Sinica, Beijing, China.

Qiang Lu (SM'85-F'03) graduated form the Graduate School of Tsinghua University, Beijing, China, in 1963.

Currently, he is a Professor at Tsinghua University and an Academician of the Chinese Academy of Science since 1991. He was a Visiting Scholar and a Visiting Professor at Washington University, St. Louis, MO, and Colorado State University, Ft. Collins, from 1984 to 1986. He was also a Visiting Professor at Kyushu Institute of Technology (KIT), Japan, from 1993 to 1995. His research interest is in nonlinear control theory application in power system and digital power systems. 